\title{
Predição do índice de temperatura do globo negro e umidade e do impacto das variações climáticas em galpões avícolas climatizados
}

\author{
Prediction of black globe humidity index and of the impact of climate changes on acclimatized broiler \\ houses
}

\author{
Raphaela Christina Costa Gomes ${ }^{\mathrm{I}}$ Tadayuki Yanagi Junior ${ }^{* *}$ Renato Ribeiro de Lima ${ }^{\mathrm{II}}$ \\ Sílvia de Nazaré Monteiro Yanagi ${ }^{\mathrm{II}}$ Valcimar Ferreira de Carvalho ${ }^{\mathrm{I}}$ Flávio Alves Damasceno ${ }^{\mathrm{I}}$
}

RESUMO

\begin{abstract}
Objetivou-se, com o presente trabalho, adaptar e validar um modelo para predição do indice de temperatura de globo negro e umidade (ITGU) no interior de galpões climatizados e analisar o efeito das variações climáticas sobre o ambiente térmico de produção por meio de cenários futuros. Os dados experimentais utilizados para o ajuste e validação do modelo matemático foram coletados em dois galpões avícolas climatizados, equipados com sistema de ventilação em modo túnel negativo e sistemas de resfriamento evaporativo do tipo material poroso umedecido e nebulização. Predições dos valores de ITGU foram estatisticamente iguais às observadas (teste $t, P>0,05$ ), com erro de 1,55 $\pm 1,08 \%$. Considerando o sistema analisado, observou-se que as mudanças climáticas previstas pelos cenários futuros tornarão os ambientes menos propícios à produção de frangos de corte, especialmente nas estações de primavera e verão, nas quais os valores de ITGU foram acima do limite de conforto.
\end{abstract}

Palavras-chave: conforto térmico, avicultura, instalações, mudanças climáticas.

\section{ABSTRACT}

The goal of this work was to adapt and to validate a model to predict black globe humidity index (BGHI) inside of acclimatized broiler houses, and analyzing the effect of climate changes on the thermal environment of production through the future scenarios. The experimental data used to adjust and validate of mathematical model were measured in two acclimatized broiler houses equipped with negative tunnel ventilation system coupled to pad and mist cooling systems. Prediction of BGHI values were statically equal to the observed (t test, $P>0.05$ ), with error of $1.55 \pm 1.08 \%$. Considering the system analyzed, the climate change foreseen by the future scenarios will turn the environment unsuitable for broiler chicken production, especially during spring and summer that presented BGHI values above to the comfort limit.

Key words: thermal comfort, poultry, buildings, climate change.

\section{INTRODUÇÃO}

Apesar do crescimento da avicultura, observa-se que, em climas tropicais e subtropicais, a exemplo do que ocorre no Brasil, os altos valores da temperatura e da umidade relativa do ar, principalmente na primavera e no verão, geram condições de desconforto térmico às aves, inibindo o seu desempenho produtivo e se constituindo em um dos principais problemas que afetam a sua criação em condições de confinamento (FONSECA, 1998).

As mudanças climáticas representam um desafio em manter a produção animal, visto que as aves, animais homeotérmicos, são sensíveis a pequenas alterações na temperatura do ambiente, que resultam em respostas negativas de desempenho produtivo. Como estratégia para adequar o ambiente térmico no interior do galpão às exigências das aves, a climatização é uma solução eficiente, pois proporciona

'Programa de Pós-graduação em Engenharia Agrícola (PPGEA), Departamento de Engenharia, Universidade Federal de Lavras (UFLA), 37200-000, Lavras, MG, Brasil. E-mail: yanagi@deg.ufla.br. *Autor para correspondência.

"Departamento de Ciências Exatas, UFLA, Lavras, MG, Brasil.

IIIPrograma de Pós-graduação em Recursos Hídricos em Sistemas Agrícolas (PPGRH), Departamento de Engenharia, UFLA, Lavras, MG, Brasil 
independência do clima externo, dentro de certos limites. Atingir o conforto térmico no interior dessas instalações avícolas, face às condições climáticas inadequadas, torna-se um desafio, uma vez que situações extremas de calor ou frio afetam consideravelmente a produção (NÄÄS et al., 2001).

No cenário de crescimento das emissões dos gases de efeito estufa, que indicam a possibilidade de impactos climáticos significativos, os modelos climáticos computacionais sugerem que poderá ocorrer aquecimento de 4 a $6^{\circ} \mathrm{C}$ em partes do país, principalmente na Amazônia, ao final do século XXI (NOBRE, 2001). Segundo o último relatório do IPCC (2007), além da Amazônia, outras regiões do Brasil também serão afetadas e alguns modelos climáticos indicam mudanças climáticas durante os próximos 50 a 100 anos. Para a região Centro-Oeste, existe uma previsão de maiores eventos de chuva e seca, altas taxas de evaporação e veranicos com ondas de calor.

A fim de se quantificar a influência das variáveis térmicas sobre o bem-estar animal, poder-seá fazer uso dos índices de conforto térmico. Com a tendência de aquecimento global, a produção avícola vê-se negativamente afetada pelo possível aumento das temperaturas, limitando as áreas onde a produção possa ser estabelecida e renovando a procura de meios para amenizar as bruscas alterações do ambiente externo sobre o interior das instalações.

Nesse contexto, objetivou-se, com o presente trabalho, adaptar e validar um modelo matemático para predição do índice de temperatura do globo negro e umidade (ITGU) no interior de galpões avícolas climatizados, equipados com ventilação em modo túnel, e simular cenários para os períodos de 1961-1990 e futuros, considerando a tendência de aquecimento para a cidade de Goiânia, GO.

\section{MATERIAL E MÉTODOS}

Os dados experimentais utilizados nesta pesquisa foram coletados em dois galpões comerciais climatizados (Sistema de Ventilação Túnel com resfriamento por meio de placas de celulose e nebulização - $S V T_{\text {PC-NEB }}$ - e Sistema de Ventilação Túnel com resfriamento por meio de painéis de sombrite e nebulização - $\mathrm{SVT}_{\mathrm{PS} \text {-NEB }}$ ), localizados no município de Itaberaí, GO (16 $01^{\circ}$ 'S de latitude, $49^{\circ} 48^{\prime}$ 'W de longitude, $722 \mathrm{~m}$ de altitude e pressão atmosférica, medida no local, de $929 \mathrm{hPa}$ ). A coleta dos dados ocorreu entre 21 e 43 dias de vida das aves, na qual os sistemas de ventilação e resfriamento evaporativo atuavam de forma efetiva, estando quatro exaustores e os sistemas de resfriamento por placa porosa umedecida ou painéis de sombrite funcionando constantemente de 10 às $18 \mathrm{~h}$ e, para temperaturas do ar acima de $29,5^{\circ} \mathrm{C}$, os sistemas acionavam o segundo estágio de exaustores, totalizando dez exaustores, associados à nebulização. Nos galpões $\mathrm{SVT}_{\mathrm{PC} \text {-NEB }}$ e $\mathrm{SVT}_{\mathrm{PS}-\mathrm{NEB}}$, foram alojadas fêmeas da linhagem Cobb, com densidades de 14 e 12,64 aves $\mathrm{m}^{-2}$, respectivamente.

\section{Galpões experimentais}

O galpão $\mathrm{SVT}_{\mathrm{PC}-\mathrm{NEB}}$ caracteriza-se por ventilação em modo túnel e sistema de resfriamento evaporativo com placa porosa de celulose $(10,70 \times 1,80 \mathrm{~m}), 16$ linhas de nebulização interna distribuídas ao longo do galpão com 89 bicos nebulizadores $\left(1,20 \pm 1,02 \mathrm{~mL} \mathrm{~s}^{-1}\right)$. O galpão $\mathrm{SVT}_{\mathrm{PS} \text { NEB }}$ é caracterizado por ventilação em modo túnel e sistema de resfriamento com dois painéis cobertos por sombrite e umedecidos com o auxílio de 47 nebulizadores, distribuídos frontalmente às placas de celulose $(10,84 \times 2,00 \mathrm{~m})$. Um sistema de nebulização com 17 linhas transversais distribuídas ao longo do galpão, totalizando 94 bicos nebulizadores $\left(1,38 \pm 0,83 \mathrm{~mL} \mathrm{~s}^{-1}\right)$, promovia o resfriamento do ar no interior do galpão. Os sistemas $\mathrm{SVT}_{\mathrm{PC}-\mathrm{NEB}}$ e $\mathrm{SVT}_{\mathrm{PS} \text {-NEB }}$ não foram dimensionados nesta pesquisa, apresentando condições comerciais de funcionamento.

Os dois galpões, orientados no sentido leste-oeste, possuíam as seguintes dimensões: $12 \times 125$ e 13,6x125m, respectivamente, e pé-direito de 2,5m; ambos com cobertura de duas águas com telhas de cimento amianto (espessura de $0,004 \mathrm{~m}$ ), pintados externamente na cor branca e com sistema de isolamento entre a face interna e oforro (de cor amarela) composto por poliuretano expandido com $0,05 \mathrm{~m}$ de espessura; alvenarias de tijolos furados $(0,12 \times 0,20 \times 0,20 \mathrm{~m})$ nas faces leste e oeste; muretas laterais com $0,45 \mathrm{~m}$ de altura e piso de concreto coberto com cama de palha de arroz reutilizada. $O$ fechamento lateral era composto por telas de arame com malha de $0,03 \mathrm{~m}$ e cortinas laterais azuis. Os galpões possuíam, ainda, três linhas de comedouros automáticos e quatro linhas de bebedouros do tipo nipple.

Coleta de dados e instrumentação

As variáveis ambientais temperatura de bulbo seco interno $\left(\mathrm{t}_{\mathrm{bs}, \mathrm{int}}\right)$, umidade relativa interna $\left(\mathrm{UR}_{\text {int }}\right.$ ), temperatura de bulbo seco externo $\left(\mathrm{t}_{\mathrm{bs}, \mathrm{ext}}\right)$ e umidade relativa externa ( $\left(\mathrm{UR}_{\text {ext }}\right)$ foram medidas por meio de sensores/registradores (marca Hobo ${ }^{\oplus}$, modelo H8 Pro, precisão $\pm 3 \%$ ), programados para coletar os dados a cada minuto. As duas primeiras variáveis foram coletadas em três pontos centralizados em relação à largura do galpão, denominados seções, a $0,3 \mathrm{~m}$ de altura 
ao longo do comprimento na parte interna de cada galpão, cujas distâncias em relação às placas de resfriamento foram de $30,62,5$ e $95 \mathrm{~m}$. As duas últimas variáveis foram coletadas na parte externa em um ponto fixo a $0,3 \mathrm{~m}$ de altura. As velocidades do ar interno $\left(\mathrm{v}_{\mathrm{int}}\right)$ e a velocidade do ar externo $\left(v_{\text {ext }}\right)$ foram medidas com anemômetros de hélice digital (precisão de $\pm 0,03 \mathrm{~m} \mathrm{~s}^{-1}$ ) na altura dos demais sensores/registradores, ou seja, a $0,3 \mathrm{~m}$ do piso, em intervalos de uma hora, entre $10 \mathrm{e} 17 \mathrm{~h}$. As temperaturas superficiais internas dos galpões foram determinadas a partir de um termômetro infravermelho, com precisão de $\pm 1^{\circ} \mathrm{C}$, posicionado à distancia de $1 \mathrm{~m}$ de cada superfície analisada. A coleta foi feita pontualmente em seis seções distribuídas ao longo do comprimento do galpão, situadas a 20,8,41,6, $62,4,83,2,104,0,114,8$ e $125,6 \mathrm{~m}$ da cabeceira oeste do galpão.

\section{Determinação do ITGU}

O ITGU é função das temperaturas de ponto de orvalho $\left(\mathrm{T}_{\mathrm{po}}\right)$ e de globo negro $\left(\mathrm{T}_{\mathrm{gn}}\right)$, conforme equação 1, proposta por BUFFINGTON et al. (1981). No modelo matemático, a $T_{\mathrm{po}}(\mathrm{K})$ é um dado de entrada medido para um determinado estado do sistema e a $\mathrm{T}_{\mathrm{gn}}$ (K), por depender de diversas variáveis, é estimada por meio de um modelo matemático.

ITGU $=T_{\mathrm{gn}}+0,36 \mathrm{~T}_{\mathrm{po}}-330,08$

A $T_{\mathrm{gn}}$ indica os efeitos combinados da energia radiante, temperatura e velocidade do ar, constituindo um meio prático de quantificar a componente de energia radiante do ambiente. Segundo KELLY et al. (1954), a carga térmica de radiação (CTR, em $\mathrm{W} \mathrm{m}^{-2}$ ), calculada pela equação 2 , expressa a radiação incidente de diferentes regiões em torno do globo negro, sendo dependente dos materiais de construção usados e de sua geometria. A CTR também pode ser calculada pela equação 3 , obtida por meio do balanço de calor na superfície do globo; o calor ganho ou perdido por radiação no globo deve ser igual ao calor ganho ou perdido por convecção.

$$
\mathrm{CTR}=\sigma \sum_{\mathrm{i}=1}^{\mathrm{n}} \mathrm{T}_{\mathrm{i}}^{4} \mathrm{~F}_{\mathrm{i}}
$$

$\mathrm{CTR}=\sigma \mathrm{T}_{\mathrm{m}}^{4}$

Sendo que: s é a constante de StefanBoltzmann $\left(5,67 \times 10^{-8} \mathrm{~W} \mathrm{~m}^{-2} \mathrm{~K}^{-4}\right)$; Ti é a temperatura de cada seção da vizinhança do globo $(\mathrm{K}) ; \mathrm{F}_{\mathrm{i}}$ é o fator de forma de cada seção da vizinhança do globo (adimensional); $\mathrm{n}$ é o número de seções da vizinhança do globo; e $\mathrm{T}_{\mathrm{m}}$ é a temperatura média radiante (em $\mathrm{K}$ ), sendo calculada conforme a equação 4 .
$\mathrm{T}_{\mathrm{m}}=100\left[2,51 \sqrt{\mathrm{v}}\left(\mathrm{T}_{\mathrm{gn}}-\mathrm{T}_{\mathrm{bs}}\right)+\left(\frac{\mathrm{T}_{\mathrm{gn}}}{100}\right)^{4}\right]^{1 / 4}$

em que, vé a velocidade do ar $\left(\mathrm{m} \mathrm{s}^{-1}\right)$.

Substituindo a equação 4 na equação 3 e igualando-a posteriormente à equação 2 , na qual os valores das temperaturas das superfícies $\left(T_{i}\right)$ são obtidos por meio de equações empíricas, pode-se determinar o valor da $\mathrm{T}_{\mathrm{gn}}$ pelo método da bisseção.

Para galpões abertos, ou seja, com as cortinas abertas, observa-se que, em torno do globo negro, há cinco regiões bem definidas: piso sombreado, piso não sombreado, horizonte, céu frio e cobertura (OLIVEIRA, 1980). Entretanto, para galpões climatizados, as regiões em torno do globo são reduzidas à cama e superfície das aves, cortinas norte e sul, forro e cabeceiras leste e oeste, que foram negligenciadas por OLIVEIRA (1980).

Dessa forma, procedeu-se à análise de componentes principais para obter-se o peso de cada variável ambiental no ajuste das equações empíricas e indicar as de maior importância, sendo que, as equações foram ajustadas por meio do procedimento PROC REG do SAS $^{\circledast}$ (SAS, 2001), para estimativa das temperaturas superficiais da cortina norte $\left(\hat{t}_{\mathrm{s}, \mathrm{cn}}\right)$, cortina sul $\left(\hat{\mathrm{t}}_{\mathrm{s}, \mathrm{cs}}\right)$, cabeceira leste $\left(\hat{t}_{s, c l}\right)$, cabeceira oeste $\left(\hat{t}_{s, c o}\right)$, piso $\left(\hat{t}_{s, p i s o}\right)$ e forro $\left(\hat{t}_{s, \text { forro }}\right)$.

Para a predição do comportamento do ITGU para a cidade de Goiânia, GO (latitude 16²0' S, longitude $49^{\circ} 15^{\prime} \mathrm{W}$ e altitude de $741 \mathrm{~m}$ ), foram utilizados os dados das normais climatológicas no período de 1961 a 1990 (Brasil, 1992). Para a composição dos cenários futuros, adicionou-se às temperaturas do $\operatorname{ar} 1,2^{\circ} \mathrm{Ce} 2,4^{\circ} \mathrm{C}$ para $\mathrm{o}$ cenário B2 (mais otimista), nos anos de 2050 e 2100 , respectivamente, e $1,7^{\circ} \mathrm{C}$ e $3,4^{\circ} \mathrm{C}$ para a composição do cenário A2 (mais pessimista) (IPCC, 2007). Esses dois cenários foram escolhidos com base em sua descrição, sendo os mais condizentes com as condições brasileiras.

As simulações foram feitas considerando as informações do galpão $\mathrm{SVT}_{\mathrm{PC}-\mathrm{NEB}}$ utilizado para a validação do modelo. Os dados climáticos de umidade relativa, temperaturas média e máxima e pressão atmosférica foram obtidos das normais climatológicas para o município de Goiânia no período de 1961-1990 (BRASIL, 1992). Os demais dados de entrada correspondem aos usados por CARVALHO (2006), por se tratarem de experimentos conduzidos paralelamente. As simulações foram realizadas no modelo computacional desenvolvido por CARVALHO (2006), incorporando-se as equações empíricas, ajustadas neste trabalho, para estimativa das temperaturas superficiais do galpão. 


\section{RESULTADOS E DISCUSSÃO}

Após a análise de componentes principais, que determinou as variáveis que mais contribuíram para o ajuste das equações, procedeu-se à análise de regressão linear múltipla para determinar as equações para a estimativa das temperaturas superficiais $\hat{\mathrm{t}}_{\mathrm{s}, \mathrm{cn}}\left({ }^{\circ} \mathrm{C}\right)$ (equação 5), $\hat{\mathrm{t}}_{\mathrm{s}, \mathrm{ss}}\left({ }^{\circ} \mathrm{C}\right)$ (equação 6), $\hat{\mathrm{t}}_{\mathrm{s}, \text { forro }}\left({ }^{\circ} \mathrm{C}\right.$ ) (equação 7), $\hat{\mathrm{t}}_{\mathrm{s}, \mathrm{ll}}\left({ }^{\circ} \mathrm{C}\right)$ (equação 8 ), $\hat{\mathrm{t}}_{\mathrm{s}, \mathrm{co}}\left({ }^{\circ} \mathrm{C}\right)$ (equação 9 ) em torno do globo negro em função das variáveis ambientais e seus respectivos coeficientes de determinação. Os coeficientes de determinação para as equações citadas previamente foram de 40,9, 56,4, 58,3, 84,3 e 72,9\%, respectivamente.

$\hat{\mathrm{t}}_{\mathrm{s}, \mathrm{cn}}=35,7-0,0324 \mathrm{UR}_{\mathrm{int}}-0,0601$

$\mathrm{UR}_{\mathrm{ext}}-0,424 \mathrm{t}+0,201 \mathrm{t}_{\mathrm{bs}, \text { ext }}$

$\hat{\mathrm{t}}_{\mathrm{s}, \mathrm{cs}}=15,5+0,265 \mathrm{t}_{\mathrm{bs}, \text { ext }}-0,359 \mathrm{t}_{\mathrm{bs}, \text { int }}+$

$0,229 \mathrm{t}+0,0261 \mathrm{UR}_{\text {int }}-0,0520 \mathrm{UR}_{\mathrm{ext}}$

$\hat{\mathrm{t}}_{\text {s. forro }}=22+0,155 \mathrm{t}_{\mathrm{bs}, \text { ext }}-0,371 \mathrm{t}+$

$0,454 \mathrm{t}_{\mathrm{bs}, \text { int }}+0,0324 \mathrm{UR}_{\mathrm{int}}-0,0938 \mathrm{UR}_{\mathrm{ext}}$

$\hat{\mathrm{t}}_{\mathrm{s}, \mathrm{cl}}=6,58-0,0321 \mathrm{UR}_{\mathrm{ext}}+0,716 \mathrm{t}+0,460 \mathrm{t}_{\mathrm{bs}, \text { int }}$

$\hat{\mathrm{t}}_{\mathrm{s}, \mathrm{co}}=167+0,170 \mathrm{t}+0,259 \mathrm{t}_{\mathrm{bs}, \text { int }}$

$-2,75 \mathrm{t}_{\mathrm{bs}, \text { ext }}+87,3 \mathrm{t}_{\mathrm{bs}, \text { ext }}, 33$

sendo, $\mathrm{UR}_{\text {int }}$ : umidade relativa interna (\%); $\mathrm{UR}_{\text {ext }}$ : umidade relativa externa $(\%)$; t: tempo $(\mathrm{h}) ; \mathrm{t}_{\mathrm{bs}, \mathrm{ext}}$ : temperatura de bulbo seco externa $\left({ }^{\circ} \mathrm{C}\right) ; \mathrm{t}_{\mathrm{bs} \text {, int }}$ : temperatura de bulbo seco interna $\left({ }^{\circ} \mathrm{C}\right)$.

Para a estimativa da $\hat{\mathrm{t}}_{\mathrm{s}, \mathrm{pis}}$, inicialmente foram ajustadas as equações para estimar as temperaturas superficiais da cama $\left(\hat{\mathrm{t}}_{\text {s.cama, }}{ }^{\circ} \mathrm{C}\right)$ (Equação 10) e das aves $\left(\hat{\mathrm{t}}_{\text {s,aves }},{ }^{\circ} \mathrm{C}\right)$ (Equação 11), separadamente, cujos coeficientes foram significativos (Teste $t, \mathrm{P}<0,05$ ).

$\hat{\mathrm{t}}_{\mathrm{s}, \text { cama }}=39,1-0,287 \mathrm{t}_{\mathrm{bs} \text {, int }}$

$\hat{\mathrm{t}}_{\mathrm{s} \text {,aves }}=19,8+0,425 \mathrm{t}$ bs, int

Para se obter a equação para estimar a $\hat{t}_{\mathrm{s}, \text { piso, }}$, que engloba as temperaturas superficiais $\hat{t}_{\mathrm{s}, \mathrm{cama}} \mathrm{e} \hat{\mathrm{t}}_{\mathrm{s}, \mathrm{aves}}$, definiu-se o parâmetro $\beta$, que corresponde à percentagem de área ocupada por aves dentro do galpão e que funciona como moderador de influência de cada região para a temperatura da superfície do piso, conforme está demonstrado na equação 12 .

$\hat{\mathrm{t}}_{\mathrm{s}, \text { piso }}=\beta \hat{\mathrm{t}}_{\mathrm{s}, \mathrm{cama}}+(1-\beta) \hat{\mathrm{t}}_{\mathrm{s}, \text { aves }}$

Substituindo-se, na equação 12 , as equações 10 e 11, tem-se a equação para a estimativa da $\hat{t}_{\text {s,piso }}\left({ }^{\circ} \mathrm{C}\right)$ (equação 13), em função apenas da $t_{b s, i n t}$.

$\hat{\mathrm{t}}_{\mathrm{s}, \text { piso }}=(\beta / 100)\left(39,1-10,287 \mathrm{t}_{\mathrm{bs}, \text { int }}\right)$ em que, $ß$ é um parâmetro que corresponde à percentagem de área ocupada pelas aves $(ß=90 \%$, valor adotado neste trabalho).

Os erros absolutos médios e os desvios padrões para os valores das temperaturas das superfícies observadas e estimadas pelas equações ajustadas são de 1,17 e 1,05, 0,84 e 0,66, 0,66 e 0,52, 0,62 e 0,48, 1,01 e 0,77 e, 0,95 e 0,72 para $\mathrm{t}_{\mathrm{s}, \mathrm{cn}}, \mathrm{t}_{\mathrm{s}, \mathrm{cs}}, \mathrm{t}_{\mathrm{s}, \mathrm{c}}, \mathrm{t}_{\mathrm{s}, \mathrm{co}}$, $t_{s, \text { piso }}$ e $t_{s, \text { forro }}$, respectivamente. Tendo em vista que os erros médios absolutos calculados entre os valores das temperaturas superficiais estimados e observados são menores ou iguais a $1,17^{\circ} \mathrm{C}$, e que a magnitude desses erros teria pouca influência sobre o resultado final para o cálculo do ITGU, pode-se considerar que as equações ajustadas são adequadas para o uso proposto.

Os valores de ITGU observados e simulados pelo modelo adaptado são ilustrados na figura 1, bem como a equação para estimativa do ITGU, que apresentou coeficientes linear e angular significativos (teste t, $\mathrm{P}<0,05$ ) e $\mathrm{R}^{2}$ de 0,4128 . Os valores médios e os desvios padrões dos ITGUs simulados e observados foram de 77,36 $\pm 1,34$ e 76,64 $\pm 1,55$, respectivamente; sendo que os valores médios do desvio absoluto e erro percentual foram de $1,18 \pm 0,81^{\circ} \mathrm{C}$ e $1,55 \pm 1,08 \%$, respectivamente. Esses resultados mostram-se promissores, tendo em vista que o sistema modelado depende de um número expressivo de variáveis sujeitas a variações expressivas e aleatórias. Outros modelos aplicados à ambiência animal têm apresentado erros próximos aos encontrados nesta pesquisa, variando de 0,65\% PONCIANO (2011) a 1,02\% LOPES (2009), ajustando redes neurais artificiais e modelos empíricos, respectivamente. Os resultados das simulações de ITGU, aplicando as equações de 1 a 9 e 13, em função das médias das temperaturas máximas do ar para o município de Goiânia, GO, usando o cenário 1961-1990 e os cenários futuros A2 e B2 (IPCC, 2007), estão ilustrados na figura 2 , que indica o aumento gradativo do ITGU ao longo dos anos dentro das instalações.

Nos meses de fevereiro (Figuras $2 \mathrm{a}$ e $2 \mathrm{~b}$ ) e novembro (Figuras $2 \mathrm{~g}$ e $2 \mathrm{~h}$ ), representando as estações de verão e primavera, todos os cenários simulados apresentaram ITGUs acima do limite de conforto indicado por MEDEIROS et al. (2005), ou seja, ITGUs no intervalo de 78 a 88, havendo redução no ganho de peso diário e diminuição de $43 \%$ na ingestão de alimentos, podendo ser considerados os piores meses para a produção avícola. As simulações para o período de 1961-1990 indicam a necessidade de se redimensionar os sistemas de climatização dos galpões, visando à adequação do ambiente térmico interno às necessidades das aves. 


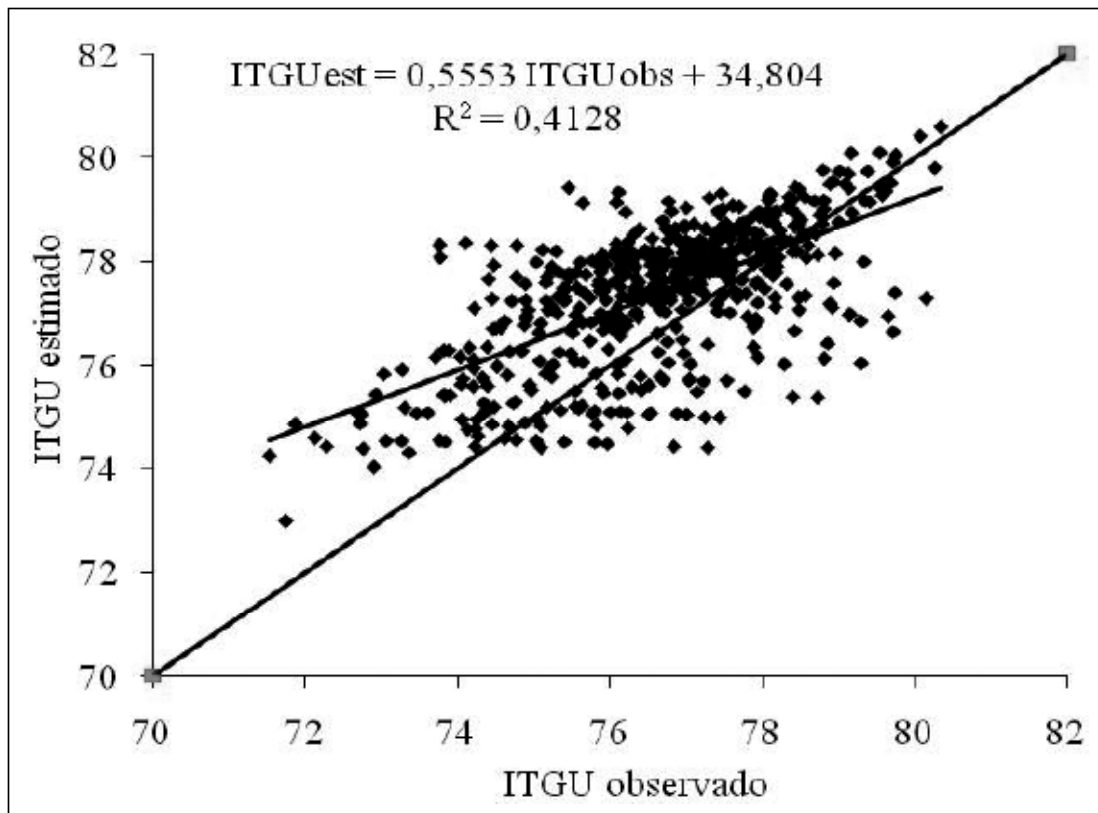

Figura 1 - Relação funcional entre os ITGUs observados e simulados pelo modelo.

O mês de maio (Figura 2c e 2d) apresenta condições de conforto em apenas $37,6 \%$ do galpão no cenário 1961-1990 e nos demais cenários todo o galpão encontra-se em desconforto térmico. Para o mês de agosto, verificou-se que $87,2 \%$ da área do galpão se encontra em condição de conforto no cenário 19611990, reduzindo bruscamente, no ano de 2050 (cenário B2), para apenas $7,2 \%$. O aumento das temperaturas superficiais das regiões em torno do globo promove a elevação da sua temperatura e, consequentemente, do ITGU, conforme constatado por FURTADO et al. (2003) ao analisar o conforto térmico em galpões avícolas.

As simulações realizadas apresentam tendências gerais que podem ser importantes na definição de estratégias de projetos de galpões e de sistemas para climatização, entretanto, condições mais críticas podem ocorrer. Ademais, devido às características de projeto do próprio galpão simulado, os resultados podem ser melhores caso ocorra o redimensionamento de forma adequada dos sistemas de ventilação e resfriamento evaporativo usados.

Em concordância com BORGES et al. (2008), os resultados desta pesquisa evidenciam os efeitos negativos do aumento da temperatura terrestre sobre o ambiente térmico de produção de frangos de corte, sendo que aqueles meses considerados como mais propícios à produção no período de 1961-1990 tendem a ser reclassificados como causadores de desconforto térmico nos cenários futuros.
Avaliando as projeções de mudanças climáticas, verificam-se impactos negativos expressivos na pecuária (MELADO, 2007; NÄÄS et al., 2010; SILVA et al., 2010) e na avicultura de corte (BORGES et al., 2008). O aquecimento do ambiente prejudica as respostas produtivas e reprodutivas, as condições metabólicas e de saúde e as respostas imunológicas dos animais (NARDONE et al., 2010), além da qualidade da carne (GREGORY, 2010). Esses resultados poderão ser danosos à economia brasileira, pois o Brasil é um dos países mais sujeitos aos impactos das mudanças climáticas (FEARNSIDE, 2009).

\section{CONCLUSÃO}

Os valores de índice de temperatura do globo negro e umidade (ITGU) simulados pelo modelo foram semelhantes aos observados na prática. $\mathrm{O}$ aumento das temperaturas do ar previsto pelos cenários futuros afetará negativamente a avicultura de corte na região em torno de Goiânia - GO - principalmente na primavera e verão.

\section{AGRADECIMENTOS}

Os autores agradecem à Coordenação de Aperfeiçoamento de Pessoal de Nível Superior (CAPES), ao Conselho Nacional de Desenvolvimento Científico e Tecnológico (CNPq) e à Fundação de Amparo à Pesquisa do Estado de Minas Gerais (FAPEMIG), pelo apoio financeiro e pelas bolsas de estudo e pesquisa aos autores. 
a.

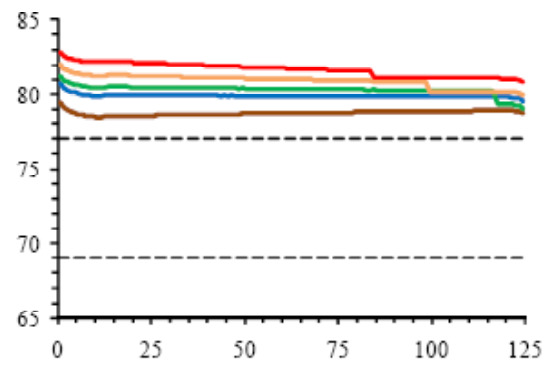

c.
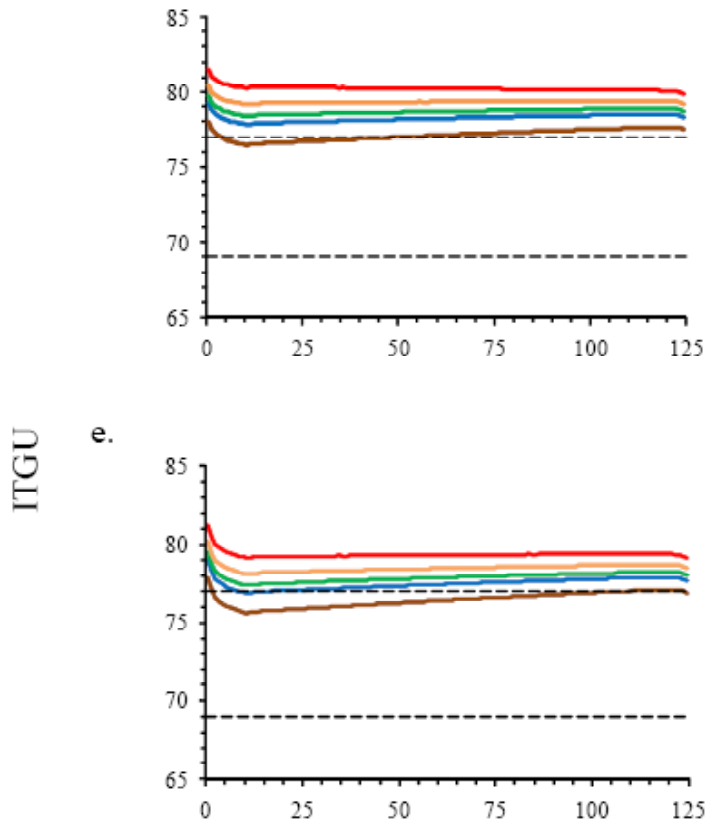

g.
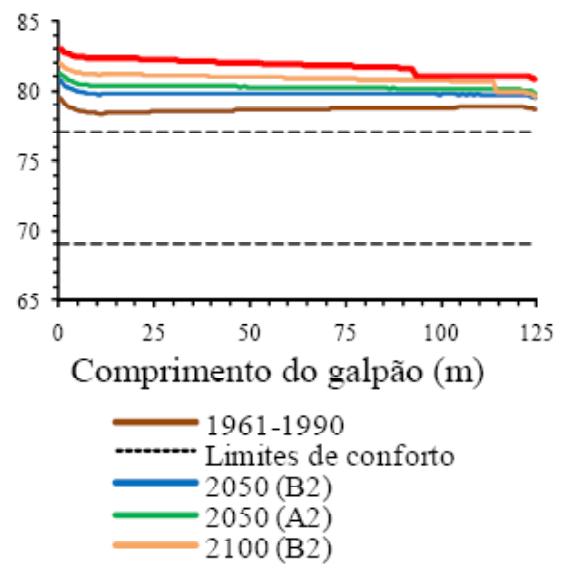

b.

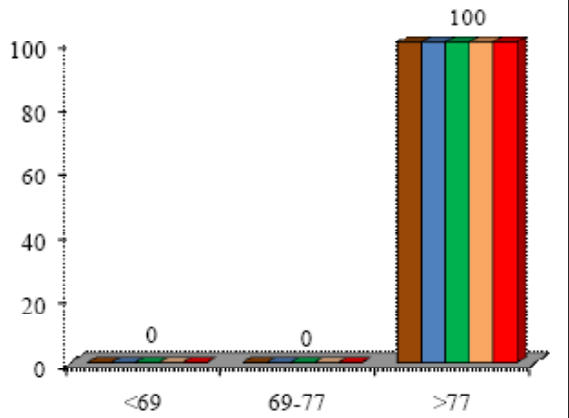

d.

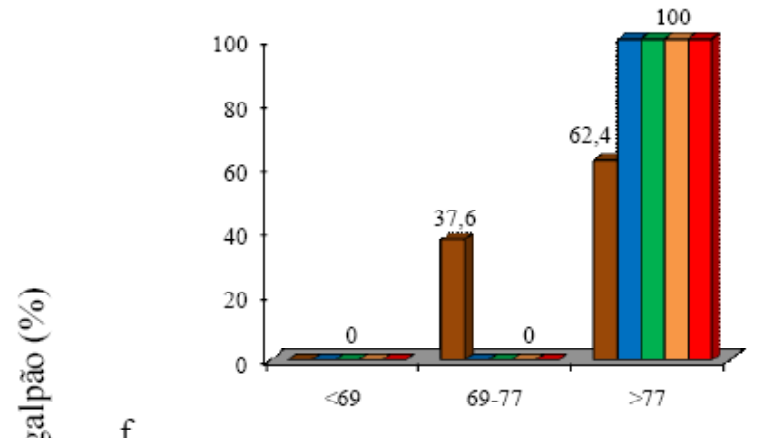

f.

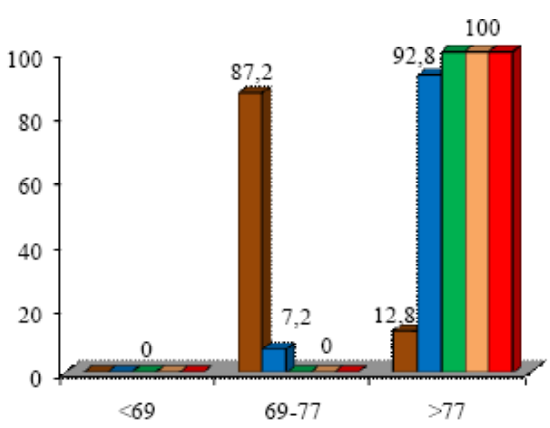

h.

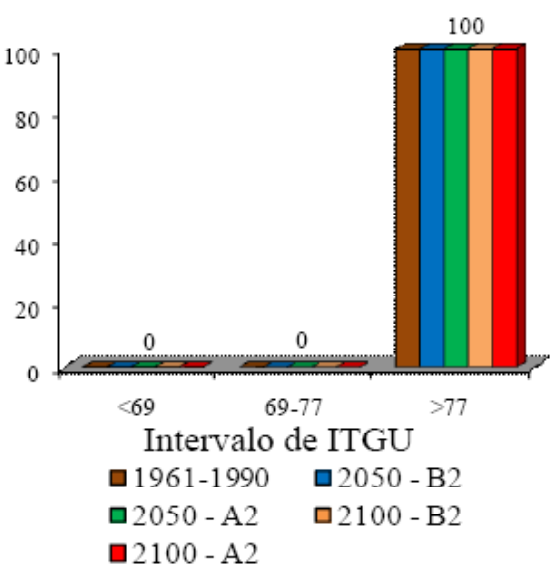

Figura 2 - Representação dos comportamentos e percentagem de área do galpão dos índices de temperatura do globo negro e umidade (ITGU), estimado em função da temperatura máxima do ar, para os meses de fevereiro (a) e (b), maio (c) e (d), agosto (e) e (f) e novembro (g) e (h), para o período de 1961-1990 e de acordo com os cenários B2 e A2 (IPCC, 2007), para os anos de 2050 e 2100. 


\section{REFERÊNCIAS}

BORGES, G. et al. Climate changes influence on inside thermal environment of broiler houses. In: INTERNATIONAL CONFERENCE OF AGRICULTURAL ENGINEERING, BRASILIAN CONGRESS OF AGRICULTURAL ENGENEERING, 37.; INTERNATIONAL LIVESTOCK ENVIRONMENT SYMPOSIUM - ILES, 8., 2008, Foz do Iguaçu, PR. Anais... Foz do Iguaçu: SBEA/ASABE/CIGR, 2008. CD-ROM.

BRASIL. Ministério da Agricultura. Normais climatológicas 1961-1990. Brasília, DF, 1992. 84p.

BUFFINGTON, C.S. et al. Black globe humidity index (BGHI) as comfort equation for dairy cows. Transactions of the ASAE, St. Joseph, v.24, n.3, p.711-714, 1981.

CARVALHO, V.F. Modelagem e zoneamento do ambiente térmico para avaliar o desempenho de frangos de corte criados em galpões climatizados. 2006. 77f. Dissertação (Mestrado em Construções Rurais e Ambiência) - Universidade Federal de Lavras, Lavras, MG.

FEARNSIDE, P.M. Global warming in Amazonia: impacts and mitigation. Acta Amazônica, Manaus, v.39, n.4, p.10031012, 2009. Disponível em: <http://www.scielo.br/ scielo.php? script $=$ sci_arttext \& pid = S 0044 $59672009000400030 \& \operatorname{lng}=e n \& n r m=i s o \& t \operatorname{lng}=e n>$. Acesso em: 29 jun. 2011. doi: 10.1590/S0044-59672009000400030.

FONSECA, J.M. Efeito da densidade de alojamento sobre o desempenho de frangos de corte criados em sistema de nebulização e ventilação em túnel. 1998. 57f. Dissertação (Mestrado em Engenharia Agrícola - Construções Rurais e Ambiência) - Universidade Federal de Viçosa, Viçosa, MG.

FURTADO, D.A. et al. Análise do conforto térmico em galpões avícolas com diferentes sistemas de acondicionamento. Revista Brasileira de Engenharia Agrícola e Ambiental, Campina Grande, v.7, n.3, p.559-564, 2003. Disponível em: <http:// www.scielo.br/scielo.php? script $=$ sci_arttext $\&$ pid $=S 1415$ $43662003000300025 \& \operatorname{lng}=$ en $\& n r m=i s o \& \operatorname{lng}=p t>$. Acesso em: 29 jun. 2011. doi: 10.1590/S1415-43662003000300025.

GREGORY, N.G. How climatic changes could affect meat quality. Food Research International, Amsterdam, v.43, n.7, p.18661873, 2010. Disponível em: <http://www.sciencedirect.com/ science/article/pii/S0963996909001707>. Acesso em: 29 jun. 2011. doi: 10.1016/j.foodres.2009.05.018.

INTERGOVERNMENTAL PANEL IN CLIMATE CHANGE. Climate change 2007: the physical science basis: summary for policymakers: Contribution of Working Group I to the Fourth Assessment Report of the Intergovernmental Panel on Climate Change. Genebra, 2007. 18p.

KELLY, C.F. et al. Design of livestock shades: construction and location of shades contribute to animal comfort and maintenance of feed intake. California Agriculture, Berkley, v.8, n.8, p.3-4, 1954.
LOPES, A.Z. Desenvolvimento de um neuro-controlador para galpões climatizados de frangos de corte. 2009. 140f. Dissertação (Mestrado em Engenharia de Sistemas, Modelagem e Análise de Sistemas) - Universidade Federal de Lavras. Lavras, MG.

MEDEIROS, C.M. et al. Efeitos da temperatura, umidade relativa e velocidade do ar em frangos de corte. Engenharia na Agricultura, Viçosa, v.13, n.4, p.277-286, 2005. Disponível em: <http://www.ufv.br/dea/reveng/arquivos/Vol13/ v13n4p277-286.pdf>. Acesso em: 29 jun. 2011.

MELADO, J. Pastagem ecológica e serviços ambientais da pecuária sustentável. Revista Brasileira de Agroecologia, Santa Maria, v.2, n.2, p.1777-1783, 2007. Disponível em: <http://www.aba-agroecologia.org.br/ojs2/index.php/ rbagroecologia/article/view/7472/5418>. Acesso em: 29 jun. 2011.

NÄÄS, I.A. et al. Controle e sistematização em ambientes de produção. In: SILVA, I.J.O. (Ed.). Ambiência na produção de aves em clima tropical. Piracicaba: NUPEA-ESALQ/ USP, 2001. V.1, p.75-148, 165p.

NÄÄS, I.A. et al. Impact of global warming on beef cattle production cost in Brazil. Scientia Agricola, Piracicaba, SP, v.67, n.1, p.1-8, 2010. Disponível em: <http://www.scielo.br/ scielo.php? script $=$ sci_arttext \& pid = S $0103-$ $90162010000100001 \& \operatorname{lng}=e^{2} \& n r m=i s o>$. Acesso em: 29 jun. 2011. doi: 10.1590/S0103-90162010000100001.

NARDONE, A. et al. Effects of climate changes on animal production and sustainability of livestock systems. Livestock Science, Amsterdam, v.130, n.1-3, p.57-69, 2010. Disponível em: <http://www.mendeley.com/research/effects-climatechanges-animal-production-sustainability-livestock-systems13>. Acesso em: 29 jun. 2011. doi: 10.1016/j.livsci.2010.02.011.

NOBRE, C.A. Mudanças climáticas globais: possíveis impactos nos ecossistemas do país. Parcerias Estratégicas, Brasília, n.12, p.239-258, 2001. Disponível em: <http:// homologa.ambiente.sp.gov.br/proclima/publicacoes/ publicacoes_portugues/impactos_ecossistemas_cnobre.pdf >. Acesso em: 29 jun. 2011.

OLIVEIRA, J.L. Hot weather livestock housing analysis. 1980. 120f. Thesis (Ph.D. in Agricultural Engineering) Michigan State University, East Lansing.

PONCIANO, P.F. Predição do desempenho produtivo e temperatura retal de frangos de corte durante os primeiros 21 dias de vida. 2011. 96f. Dissertação (mestrado em Engenharia Agrícola) - Universidade Federal de Lavras, MG.

SAS INSTITUTE. User's guide: release 8.2. Cary, 2001. $943 \mathrm{p}$.

SILVA, T.G.F. et al. Cenários de mudanças climáticas e seus impactos na produção leiteira em estados nordestinos. Revista Brasileira de Engenharia Agrícola e Ambiental, Campina Grande, v.14, n.8, p.863-870, 2010. Disponível em: <http:// www.scielo.br/pdf/rbeaa/v14n8/v14n08a11.pdf >. Acesso em: 29 jun. 2011. 